\title{
Research Progress in Graphene as Sulfur Hosts in Lithium-Sulfur Batteries
}

\author{
Mengdi Zhang, Bei Chen, Mingbo Wu * \\ State Key Laboratory of Heavy Oil Processing, College of New Energy, College of Chemical Engineering, China University of \\ Petroleum (East China), Qingdao, 266580, Shandong Province, China.
}

\begin{abstract}
Lithium-sulfur batteries are considered to be one of the most promising new-generation energy storage devices, owing to their ultra-high theoretical energy density and the merits of sulfur cathodes, which include natural abundance, low cost, and no toxicity. However, the commercial application of lithium-sulfur batteries is still subject to various intractable challenges. First, the insulation of sulfur and its solid discharge products $\left(\mathrm{Li}_{2} \mathrm{~S}_{2} / \mathrm{Li}_{2} \mathrm{~S}\right)$ leads to low utilization of the active materials. Second, the cathode suffers from an $80 \%$ volume expansion after the discharge process, which adversely affects its structural stability. Finally, intermediary lithium polysulfides can easily dissolve into the electrolyte, which can trigger the "shuttle effect." This results in the loss of active materials, fast capacity fading, and low Coulombic efficiency. Graphene has garnered significant interest as a host material to accommodate sulfur for high-performance lithium-sulfur battery. A graphene host

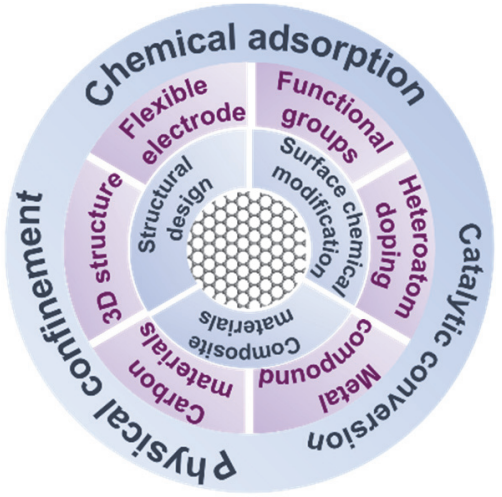
featuring a high specific surface area, excellent conductivity, and excellent mechanical stability can ensure a good electrical contact between the sulfur species and the current collector and withstand the volumetric strain of the electrode during cycling. Unfortunately, lithium polysulfides are still prone to escape from cathodes owing to the open two-dimensional (2D) plane structure of graphene sheets. To address this issue, various graphene-based materials with unique structures and chemical compositions have been trialed as sulfur hosts. In this review, we summarize research progress regarding threedimensional (3D) graphene, graphene with modified surface chemistry, graphene-based composites, and graphene-based flexible materials as sulfur hosts for lithium-sulfur batteries. Furthermore, we analyze the challenges of applying graphene host materials in high-performance lithium-sulfur batteries. This review is mainly divided into four parts: (1) 3D graphene materials as sulfur hosts: the interconnected 3D porous network structure assembled from 2D graphene sheets provides a half-enclosed cavity to accommodate sulfur and its discharge products, which can inhibit the diffusion of lithium polysulfides to a certain extent. (2) Graphene materials with modified surface chemistry as sulfur hosts: hydrophilic surface functional groups and doped non-metal or metal heteroatoms on graphene can chemically adsorb polar lithium polysulfides. (3) Graphene-based composites as sulfur hosts: in various graphene-based composites, graphene usually functions as a conductive and flexible substrate. Other components, such as other types of carbon or metal compounds, can play an important role in restricting lithium polysulfides and propelling their reaction kinetics. (4) Flexible graphene-sulfur electrodes: the excellent flexibility and conductivity of graphene endowed it and its composites with a broad range of prospective applications regarding flexible lithium-sulfur batteries.
\end{abstract}

Key Words: Lithium-sulfur battery; Sulfur host; Graphene; Graphene-based composite; Flexible cathode

Received: January 4, 2021; Revised: January 26, 2021; Accepted: January 27, 2021; Published online: February 8, 2021.

${ }^{*}$ Corresponding author. Email: wumb@upc.edu.cn.

The project was supported by the National Natural Science Foundation of China (22005341), the Shandong Provincial Natural Science Foundation

(ZR2018ZC1458, ZR2020QB128), the YanKuang Group Co., Ltd. Technology Project (YKKJ2019AJ08JG-R63), the Taishan Scholar Project (ts201712020),

and the Technological Leading Scholar of 10000 Talent Project (W03020508).

国家自然科学基金(22005341), 山东省自然科学基金(ZR2018ZC1458, ZR2020QB128), 充矿集团科技项目(YKKJ2019AJ08JG-R63), 泰山学者计划

(ts201712020)及中组部万人计划科技创新领军人才(W03020508)资助

(C) Editorial office of Acta Physico-Chimica Sinica 


\title{
石墨烯作为硫载体在锂硫电池中的研究进展
}

\author{
张梦迪, 陈蓓, 吴明铂 ${ }^{*}$ \\ 重质油国家重点实验室，中国石油大学(华东)新能源学院，化学工程学院，山东 青岛 266580
}

\begin{abstract}
摘要: 锂硫电池因其超高的理论能量密度以及硫资源丰富、成本低廉、无毒的优点, 被认为是极具发展潜力与应用前景 的新一代储能设备。然而, 硫正极导电性差、体积膨胀以及穿梭效应严重等问题严重制约了其商业化应用。石墨烯具有 高比表面积、高导电性和高柔㓞性, 并且易于进行表面化学修饰及组装, 是一种理想的硫载体材料。本文主要综述了近 年来三维石墨烯、表面化学修饰的石墨烯、石墨烯基复合材料以及石墨烯基柔性材料在锂硫电池正极中的研究现状, 并 展望了石墨烯作为硫载体在锂硫电池正极中的发展趋势。
\end{abstract}

关键词：锂硫电池；硫载体；石墨烯；石墨烯基复合材料；柔性正极 中图分类号: 0646

\section{1 引言}

随着新能源的大规模开发与使用、电动汽车 以及智能电网的快速发展, 开发高性能、低成本的 电化学储能体系势在必行 1,2 。锂硫电池理论能量 密度高达 $2600 \mathrm{Wh} \cdot \mathrm{kg}^{-1}$, 是目前商业化锂离子电池 能量密度的 5 倍以上。而且, 硫还具有储量丰富、 成本低廉、无毒的优点。因此, 锂硫电池被认为是 极具发展潜力与应用前景的新一代电池体系 3,4 。

锂硫电池的工作原理如图 $1 \mathrm{a}$ 所示 ${ }^{5}$ 。在放电过 程中, 负极锂失去电子生成锂离子, 正极硫接收通 过外电路传递来的电子并与锂离子结合生成 $\mathrm{Li}_{2} \mathrm{~S}$ 。 充电过程与之相反。实际上, 在充放电过程中, 锂 硫电池正极经历了一个复杂的多电子反应过程。 如图 $1 \mathrm{~b}$ 所示, 放电时, 环状的 $\mathrm{S}_{8}$ 分子首先开环并与 $\mathrm{Li}^{+}$结合生成可溶性的多硫化锂 $\left(\mathrm{Li}_{2} \mathrm{~S}_{x}, 4 \leq x \leq 8\right)$, 然后继续被还原生成固态的 $\mathrm{Li}_{2} \mathrm{~S}_{2}$ 和 $\mathrm{Li}_{2} \mathrm{~S}$ 。该 “固液-固” 转化过程为正极带来了诸多挑战: (1)硫及 其放电产物 $\mathrm{Li}_{2} \mathrm{~S}_{2} / \mathrm{Li}_{2} \mathrm{~S}$ 的电子绝缘性导致活性物质 利用率较低 ${ }^{6,7}$; (2) 由硫生成 $\mathrm{Li}_{2} \mathrm{~S}$ 的过程伴随着 $80 \%$ 的体积膨胀, 严重损害了电极结构稳定性 ${ }^{8,9}$; (3)中 间产物多硫化锂易溶于电解液并在正负极之间来 回往复, 即发生所谓的 “穿梭效应”, 导致正负极活 性物质损失、电池容量衰减以及库仑效率降低 ${ }^{10-12}$ 。

优化锂硫电池正极最常用的策略是将碳基材 料作为活性物质硫的载体 ${ }^{13-15}$ 。理想的硫载体材料

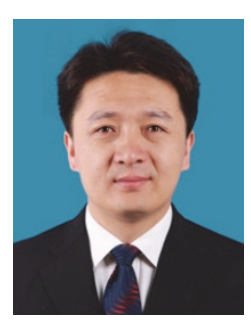

吴明铂，1972年出生。2004年获大连理 工大学博士学位, 现为中国石油大学 (华东)教授、博士生导师, 新能源学院 常务副院长。主要研究方向为新型及高 性能碳素材料的制备及其在储能领域 的应用。
应具备以下特点：(1)具有高的比表面积和发达的 孔隙结构, 能够为硫及其放电产物提供充足的储 存空间, 并且为锂离子提供快速传输通道; (2)具 有优异的导电性, 能够为活性物质提供良好的电 化学接触; (3)具有优异的结构稳定性, 能够缓解 充放电过程中的电极结构应变; (4)对多硫化锂具 有强的吸附作用以及催化转化作用, 加快正极氧 化还原反应动力学, 抑制穿梭效应。

石墨烯, 作为一种由单层 $s p^{2}$ 杂化碳原子构成 的二维碳材料, 具有高的理论比表面积 (2630 $\left.\mathrm{m}^{2} \cdot \mathrm{g}^{-1}\right)$ 、极高的本征载流子迁移率 $(200000$ $\mathrm{cm}^{2} \cdot \mathrm{V} \cdot \mathrm{s}^{-1}$ )以及优异的柔韧性和机械稳定性 ${ }^{16,17}$ 。因 此, 石墨烯作为硫载体在锂硫电池正极中具有极 大的应用潜力 ${ }^{18-20}$ 。遗憾的是, 石墨烯片层的少孔、 开放结构并不利于约束循环过程中多硫化锂的扩 散, 也就无法有效缓解穿梭效应。早期, 研究者们 主要通过对石墨烯进行三维结构设计、表面化学 修饰以及与多孔碳材料复合等策略来解决这一难

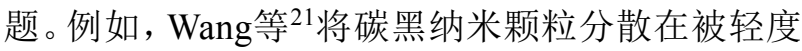
氧化的石墨烯表面。同时, 通过化学反应沉积策略 得到聚乙二醇包覆的亚微米级硫颗粒。最终, 将两 者混合分散即可得到石墨烯包覆硫颗粒的复合材 料。该材料作为锂硫电池正极, 在 $0.5 C$ 电流密度 下, 第 10 圈到第 100 圈的容量衰减率仅为 $9 \%$ 。 $\mathrm{J}$ 等 22 利用化学反应沉积方法将硫固定在二维氧化石墨 烯上, 得到了氧化石墨烯-硫复合材料(GO-S)。GO 表面丰富的含氧官能团能够有效吸附硫物种, 限 制多硫化物的溶解, 从而使得GO-S正极具有较好 的电化学性能。Yang等 ${ }^{23}$ 通过水热和化学活化方法 在石墨烯表面均匀包覆了多孔碳层, 其丰富的孔 道作为多硫化锂的 “蓄液池”，抑制了多硫化锂的 扩散, 从而提高了循环性能。石墨烯另一突出的优 
(a)

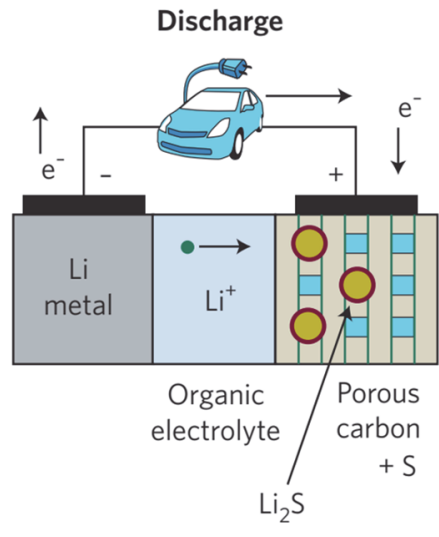

(b)

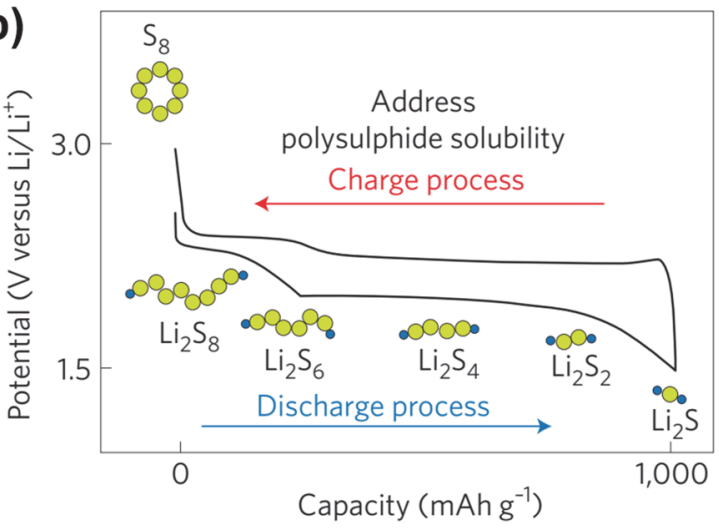

图 1 锂硫电池的(a)工作原理图和(b)正极充放电过程示意图 5

Fig. 1 (a) Schematic diagram and (b) charge/discharge process of lithium-sulfur battery ${ }^{5}$.

Adapted from Nature Publishing Group.

点是可以作为生长基底诱导各种高电化学活性材 料在其表面成核生长 ${ }^{24-27}$ 。最近, 各种石墨烯/金属 化合物复合材料被作为硫载体应用于锂硫电池正 极, 其中的极性金属化合物能够有效吸附多硫化 锂并加快其转化反应速率, 从而进一步提升锂硫电 池的电化学性能28-30。另外, 石墨烯优异的柔韧性令 其在柔性锂硫电池应用中也得到了广泛关注与研 究 31,32 。

本文主要综述了近年来三维石墨烯、表面化 学修饰的石墨烯、石墨烯基复合材料以及石墨烯 基柔性材料作为硫载体在锂硫电池正极中的研究 进展, 并展望了石墨烯载体在锂硫电池正极中的 发展趋势。

\section{2 石墨烯作为硫载体在锂硫电池中的研究 进展}

\section{1 三维石墨烯}

如前所述, 石墨烯开放的二维平面结构无法有 效约束多硫化锂的扩散, 会导致锂硫电池容量快 速衰退。而且, 石墨烯片层之间的 $\pi-\pi$ 相互作用令 石墨烯片相互堆叠, 导致其实际比表面积大大减 小, 无法完全发挥出性能优势。研究表明, 将片层 石墨烯组装成三维多孔结构, 能够为硫及其放电 产物提供相对封闭的储存空间, 一定程度上可以 抑制穿梭效应, 改善锂硫电池的电化学性能。清华 大学魏飞和张强教授团队在这方面做了很多代表 性工作。例如, 他们利用真空辅助热膨胀方法制备 了分级多孔石墨烯, 石墨烯片层之间松散堆叠弯 曲形成相互连通的三维大孔结构, 比表面积为 434 $\mathrm{m}^{2} \cdot \mathrm{g}^{-1}$ 。然后, 将硫封装在这些多孔导电网络中得到 了多孔石墨烯/硫复合材料, 在 $0.5 C$ 和 $10 C$ 下能够分 别表现出 1068 和 $543 \mathrm{mAh} \cdot \mathrm{g}^{-1}$ 的倍率容量 ${ }^{33}$ 。他们
还利用煅烧过后的介孔层状双金属氢氧化物 $(\mathrm{Mg}$ $\mathrm{Al}$ LDH)作为模板, 采用化学气相沉积(CVD)法在 其表面沉积石墨烯层, 其中部分碳被沉积在 LDH 的介孔中形成突起, 将模板去除后即可得到被大 量突起分隔的两片未堆叠石墨烯(DTG), 比表面积 高达 $1628 \mathrm{~m}^{2} \cdot \mathrm{g}^{-1}$ 。这些片层之间介孔尺寸的突起能 够封装多硫化锂, 抑制穿梭效应。因此, DTG与硫 的复合材料(DTG/S)表现出优良的倍率性能, 在 $5 C$ 和 $10 C$ 的大电流密度下, 初始比容量分别高达 1034 和 $734 \mathrm{mAh} \cdot \mathrm{g}^{-1}$, 循环 1000 圈后仍然保持 530 和 380 $\mathrm{mAh} \cdot \mathrm{g}^{-1}$ (图2a, b) ${ }^{34}$ 。另一工作是以 $\mathrm{CaO}$ 作为模板, 通过 CVD法制备了比表面积为 $572 \mathrm{~m}^{2} \cdot \mathrm{g}^{-1}$ 的分级 多孔石墨烯(HPG)。石墨烯表面分布着丰富的平面 微孔, 能够限制多硫化锂扩散; 片层弯曲褶皱形成 了大量介孔, 有利于离子的快速传输和多硫化锂 的限域; 片层之间构建了大孔尺寸的空腔, 能够进 一步为锂离子传输提供畅通通道。将 $\mathrm{HPG}$ 与硫复 合并用于锂硫电池正极, 在 $5 C$ 大电流密度下表现 出 $357 \mathrm{mAh} \cdot \mathrm{g}^{-1}$ 的比容量(图2c, d) ${ }^{35}$ 。

\section{2 表面化学修饰的石墨烯}

极性多硫化锂与非极性石墨烯之间的相互作 用较弱, 因而仅依靠空间限域并不能有效阻止多 硫化锂从石墨烯基底逃逸。研究者们发现, 通过表 面化学修饰可以提高石墨烯基底的极性, 从而对 多硫化锂产生较强的化学吸附作用。

\subsection{1 官能化石墨烯}

在石墨烯表面嫁接亲水性官能团有利于多硫 化锂在石墨烯基底上针定。Zu等 ${ }^{36}$ 首先通过水热方 法在石墨烯表面均匀生长了丰富的羟基基团, 然 后以羟基作为硫的成核位点, 通过氢键作用将化 学反应得到的无定型硫均匀负载在羟基化石墨烯 表面。硫如此高的分散状态使其与导电基底充分 
(a)

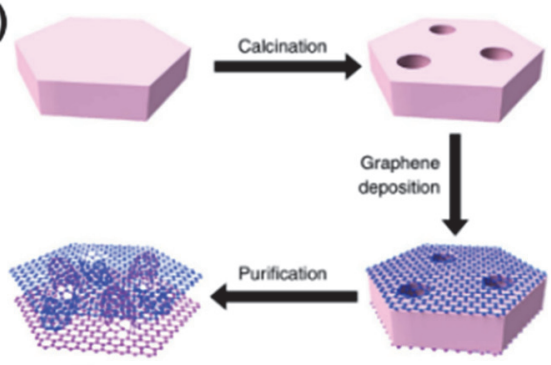

(c)

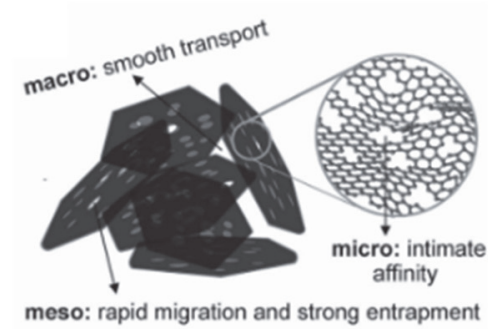

(b)

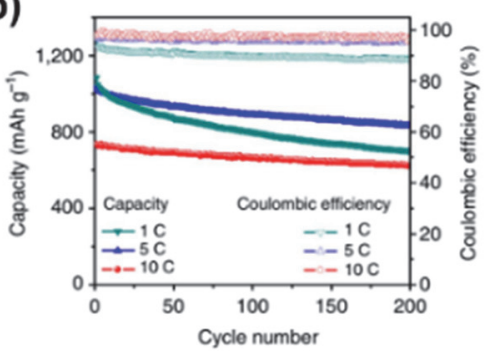

(d)

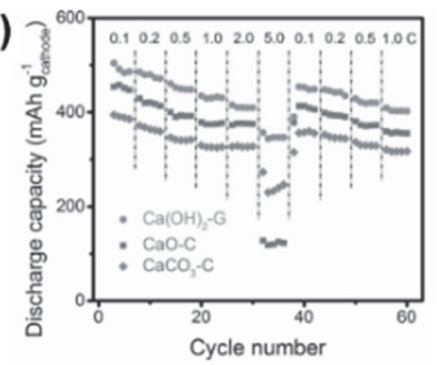

图 2 (a) DTG 的合成示意图; (b) DTG/S 正极的循环性能 ${ }^{34}$; (c) HPG 的孔结构图示图; (d) HPG/S 正极的电化学性能 ${ }^{35}$

Fig. 2 (a) Schematic for the synthesis of unstacked DTG; (b) Cycling performance of the DTG/S cathode ${ }^{34}$;

(c) Illustration for the pore structure of HPG; (d) Rate performance of HPG/S cathode ${ }^{35}$.

Adapted from Nature Publishing Group and Wiley publisher, respectively.

接触, 从而表现出优异的倍率性能。在 $2 C$ 大电流 密度下, 初始比容量高达 $647 \mathrm{mAh} \cdot \mathrm{g}^{-1}$, 循环 100 圈 后仍然保持 $647 \mathrm{mAh} \cdot \mathrm{g}^{-1}$ 。Chang等 37 通过一锅法制 备了功能化多孔石墨烯/硫复合材料。石墨烯表面 被 $\mathrm{H}_{2} \mathrm{O}_{2}$ 氧化刻蚀形成了丰富的微孔, 同时沿着孔 边缘掺杂了丰富的含氧官能团, 这些极性含氧官 能团对多硫化锂具有强的针定作用。该正极材料 在 $0.2 C$ 电流密度下循环 300 圈后, 比容量保持 429 $\mathrm{mAh} \cdot \mathrm{g}^{-1}$ 。Wang等 ${ }^{38}$ 通过在石墨烯表面嫁接乙二胺 官能团有效抑制了固体放电产物从石墨烯基底脱 离。理论计算证明, $\mathrm{Li}_{2} \mathrm{~S}$ 中的锂原子能和乙二胺中 的氮原子形成共价键, 从而增强其与石墨烯基底 之间的结合能(图3)。将乙二胺功能化石墨烯与硫 (a)

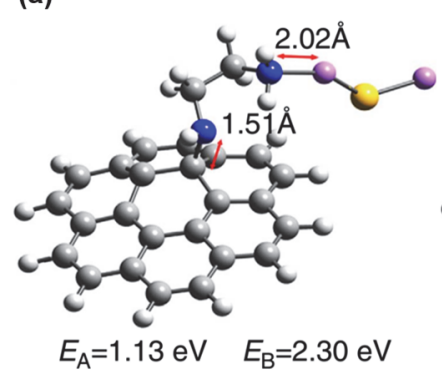

(b)

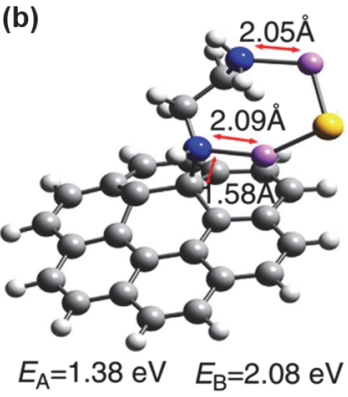

\section{OC $\supset \mathrm{H} O \mathrm{~N}$ OS OLI}

图 $3 \mathrm{Li}_{2} \mathrm{~S}$ 与 $\mathrm{EFG}$ 的相互作用示意图 ${ }^{38}$

Fig. 3 The interaction between $\mathrm{Li}_{2} \mathrm{~S}$ cluster and $\mathrm{EFG}^{38}$. Adapted from Nature Publishing Group.
复合用于锂硫电池正极, 在 $0.5 C$ 电流密度下循环 350 圈后, 仍具有 $80 \%$ 的容量保持率; 在 $4 C$ 大电流 密度下仍具有 $480 \mathrm{mAh} \cdot \mathrm{g}^{-1}$ 的高比容量。

\section{2 .2 杂原子掺杂石墨烯}

除了在石墨烯表面引入官能团, 对石墨烯进 行杂原子掺杂也能加强石墨烯基底对极性多硫化 锂的吸附能力。Q Qiu等 ${ }^{39}$ 首先通过将 $\mathrm{GO}$ 在 $\mathrm{NH}_{3}$ 气氛 下热处理制备了氮掺杂石墨烯 $(\mathrm{NG})$, 然后通过化 学反应沉积在 $\mathrm{NG}$ 表面均匀负载小尺寸的硫纳米 颗粒, 得到了 $\mathrm{S} @ \mathrm{NG}$ 复合材料。 $\mathrm{NG}$ 不仅具有优异 的导电性, 而且其中的 $\mathrm{N}$ 原子可作为多硫化锂的极 性吸附位点, 有效抑制穿梭效应。因此, 该 $\mathrm{S} @ \mathrm{NG}$ 正极在不添加碳黑导电剂的情况下仍表现出良好 的电化学性能。在 $5 C$ 大电流密度下, 比容量高达 $606 \mathrm{mAh} \cdot \mathrm{g}^{-1}$, 且在 $2 C$ 下循环 2000 圈后, 容量衰退 率仅为 $0.028 \%$ 每圈。Xie等 ${ }^{40}$ 采用一步水热法制备 了三维嗍掺杂石墨烯气凝胶(BGA), 掺杂的硼原子 能够有效提高材料的导电性和对多硫化锂的吸附 能力, 将 $\mathrm{BGA}$ 作为硫载体应用于锂硫电池正极表 现出良好的电化学性能, 在 $2 C$ 电流密度下循环 200 圈后, 仍有 $600 \mathrm{mAh} \cdot \mathrm{g}^{-1}$ 的比容量。 $\mathrm{Xu}^{\text {等 }}{ }^{41}$ 将聚吡 咯官能化的石墨烯进行 $\mathrm{K}_{2} \mathrm{CO}_{3}$ 活化得到了三维氮、 硫共掺杂多孔石墨烯(A-NSG), 碳晶格中掺入的吡 啶氮和噻吩硫能够调控局域电子排布, 从而增加 石墨烯基底与多硫化锂的结合能力, 提高多硫化 锂转化的可逆性。将A-NSG 与硫复合并应用于锂 
硫电池正极, 在 $0.2 C$ 电流密度下初始比容量为 $1178 \mathrm{mAh} \cdot \mathrm{g}^{-1}$, 在 $5 C$ 大电流密度下仍保持 651 $\mathrm{mAh} \cdot \mathrm{g}^{-1}$ 。 $\mathrm{Hou}$ 等 ${ }^{42}$ 通过第一原理计算研究了不同 杂原子掺杂的石墨烯对多硫化锂的吸附作用及机 制。结果表明, 具有孤对电子的氮原子和氧原子能 够通过与多硫化锂中的锂离子之间的偶极-偶极相 互作用显著增强石墨烯基底对硫物种的吸附, 从而 实现高的比容量和库伦效率。相反, B、F、S、P 和 $\mathrm{Cl}$ 杂原子对增强石墨烯与硫物种之间的相互作 用的效果不尽人意(图4)。由此可见, 对石墨烯进 行氮掺杂、氧掺杂或者多原子掺杂是提高锂硫电 池电化学性能行之有效的方法。

\subsection{3 金属单原子修饰的石墨烯}

最新研究表明, 金属单原子修饰的石墨烯在 锂硫电池中具有广阔的应用前景。一方面, 金属单 原子具有 $100 \%$ 的原子利用率、不饱和的配位环境 和独特的电子结构, 不仅能有效吸附多硫化锂, 而 且能显著加快其氧化还原反应动力学 43-48。另一方 面, 石墨烯的二维平面结构有利于实现金属单原 子的高载量分散, 进一步提高其吸附和催化转化 能力 ${ }^{49}$ 。 $\mathrm{Du}^{\text {等 }}{ }^{50}$ 将吸附有钴离子的氧化石墨烯在氨 气气氛下高温热解制得了单原子 $\mathrm{Co}$ 修饰的 $\mathrm{N}$ 掺杂 石墨烯(Co-N/G)。DFT理论计算表明, 所形成的Co$\mathrm{N}-\mathrm{C}$ 配位结构能够有效促进放电和充电过程中 $\mathrm{Li}_{2} \mathrm{~S}$ 的形成和分解, 加快其反应动力学(图5)。在硫 面载量为 $2 \mathrm{mg} \cdot \mathrm{cm}^{-2}$ 时, 在 $0.2 C$ 电流密度下, $\mathrm{S} @ \mathrm{Co}-$ $\mathrm{N} / \mathrm{G}$ 复合材料初始比容量为 $1210 \mathrm{mAh} \cdot \mathrm{g}^{-1}$; 当硫面 载量增加到 $6 \mathrm{mg} \cdot \mathrm{cm}^{-2}$ 时, 在 $0.2 C$ 电流密度下循环

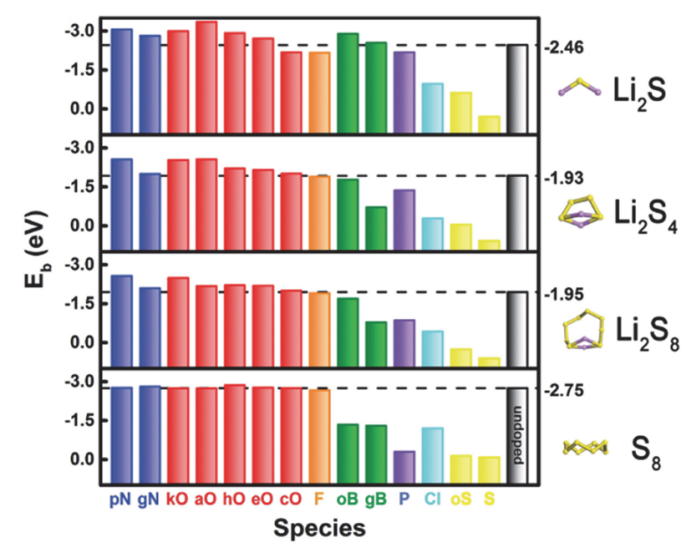

图 4 基于 DFT 计算的 $\mathrm{Li}_{2} \mathrm{~S} 、 \mathrm{Li}_{2} \mathrm{~S}_{4} 、 \mathrm{Li}_{2} \mathrm{~S}_{8}$ 和 $\mathrm{S}_{8}$ 分别与 原始石墨烯和 $\mathrm{X}$ 原子 $(\mathrm{X}=\mathrm{N} 、 \mathrm{O} 、 \mathrm{~F} 、 \mathrm{~B} 、 \mathrm{P} 、 \mathrm{Cl} 、 \mathrm{~S})$ 掺杂 的石墨烯之间的结合能 42

Fig. 4 Binding energies of $\mathrm{Li}_{2} \mathrm{~S}, \mathrm{Li}_{2} \mathrm{~S}_{4}, \mathrm{Li}_{2} \mathrm{~S}_{8}$ and $\mathrm{S}_{8}$ with $\mathrm{X}$-doped $(\mathrm{X}=\mathrm{N}, \mathrm{O}, \mathrm{F}, \mathrm{B}, \mathrm{P}, \mathrm{Cl}, \mathrm{S})$ graphene based on DFT calculations ${ }^{42}$.

Adapted from Wiley publisher.
100 圈后, 其面积比容量高达 $5.1 \mathrm{mAh} \cdot \mathrm{cm}^{-2}$ 。Wang 等 ${ }^{51}$ 将 $\mathrm{GO}$ 与 $\mathrm{Fe}(\mathrm{OH})_{3}$ 溶胶混合并在 $\mathrm{Ar} / \mathrm{H}_{2} / \mathrm{NH}_{3}$ 混合 气中高温㷽烧得到 $\mathrm{FeN}_{0.056} / \mathrm{Fe}_{3} \mathrm{C}$ 纳米颗粒嵌入的 氮掺杂石墨烯复合材料, 经过酸洗去除这些纳米 颗粒最终得到了多洞的 $\mathrm{Fe} 、 \mathrm{~N}$ 共掺杂石墨烯 $(\mathrm{HFeNG})$ 。多洞结构可以提供离子快速传输通道, 加快 $\mathrm{Li}^{+}$扩散, 从而提高电化学反应速率。 $\mathrm{Fe}$ 单原 子分别以 $\mathrm{Fe}-\mathrm{N}_{4}$ 结构针定在石墨烯面内和以 $\mathrm{Fe}-\mathrm{N}_{2}$ 配位结构位于洞的边缘处。值得注意的是, $\mathrm{Fe}-\mathrm{N}_{2}$ 配位中心与多硫化物的结合能力更强。将 $\mathrm{HFeNG}$ 载硫后用作锂硫电池的正极, 在 $5 C$ 大电流密度下, 比容量高达 $810 \mathrm{mAh} \cdot \mathrm{g}^{-1}$ 。Z Zhou等 ${ }^{52}$ 通过实验与理 论计算分析了一系列金属单原子(包括 $\mathrm{Fe} 、 \mathrm{Mn}$ 、 $\mathrm{Ru} 、 \mathrm{Co} 、 \mathrm{Zn} 、 \mathrm{~V} 、 \mathrm{Cu}$ 和 $\mathrm{Ag}$ ) 修饰的氮掺杂石墨烯对 多硫化锂的吸附能力和催化转化能力。结果发现, 除单原子 $\mathrm{Ag}$ 和 $\mathrm{Cu}$ 之外, 其他金属单原子在循环过 程中均能保持结构稳定。通过理论计算可知, $\mathrm{Li}_{2} \mathrm{~S}$ 在各种基底上的分解能垒由小到大依次排列为 $\mathrm{V}<$ $\mathrm{Mn}<\mathrm{Ru}<\mathrm{Fe}<\mathrm{Co}<\mathrm{Zn}$, 而与 $\mathrm{Li}_{2} \mathrm{~S}_{6}$ 的结合能由大 到小依次排列为 $\mathrm{V}>\mathrm{Ru}>\mathrm{Co}>\mathrm{Zn}>\mathrm{Fe}>\mathrm{Mn}$ 。综 合以上结果可知, SAV@NG对多硫化物具有最强的 吸附能力和催化转化能力。实验合成的 $\mathrm{SAV} @ \mathrm{NG} / \mathrm{S}$ 复合材料也表现出最佳的电化学性能, 在 $3 C$ 大电 流密度下, 放电比容量高达 $645 \mathrm{mAh} \cdot \mathrm{g}^{-1}$ 。
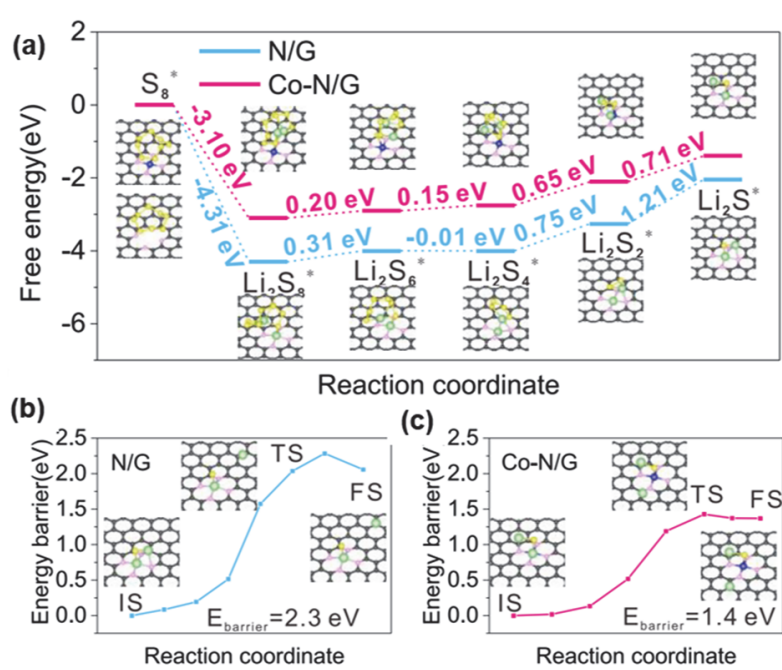

图 5 (a)硫物种在 $N / G$ 和 Co-N/G 基底上的还原反应 吉布斯自由能曲线; $\mathrm{Li}_{2} \mathrm{~S}$ 在(b) N/G 和(c) Co-N/G 上的 分解能垒曲线 50

Fig. 5 (a) Energy profiles for the reduction of sulfur species on N/G and Co-N/G substrates; Energy profiles of the decomposition of $\mathrm{Li}_{2} \mathrm{~S}$ cluster on (b) N/G and (c) $\mathrm{Co}-\mathrm{N} / \mathrm{G}^{50}$.

Adapted from American Chemical Society. 


\section{3 石墨烯基复合材料}

\subsection{1 石墨烯/碳复合材料}

通常, 单一的一种碳材料难以全部满足正极 硫载体的要求, 将具有不同优点的碳材料复合是 实现锂硫电池正极性能优化的有效策略。石墨烯 无可比拟的导电性和优异的结构稳定性令其在碳 复合材料中广受欢迎 53,54 。其他类型的碳材料, 如 多孔碳、杂原子掺杂碳等也可弥补石墨烯开放的 片层结构无法有效约束多硫化锂的缺点 ${ }^{55,56}$ 。除了 各组分的本征优势之外, 复合碳材料还可被设计 成各种独特结构, 能够进一步改善锂硫电池电化 学性能 ${ }^{57-60}$ 。Zhang等 ${ }^{61}$ 利用模板法在导电石墨烯 表面构筑了丰富的孔状/管状碳通道(NTPC-G), 这 些氮掺杂碳通道不仅为硫和固体放电产物提供了 充足的储存空间, 而且对多硫化锂起到了多重限 域作用(图6a)。因此, NTPC-G与硫的复合材料在 $6 C$ 大电流密度下, 初始比容量仍高达 $563 \mathrm{mAh} \cdot \mathrm{g}^{-1}$, 循环 600 圈之后容量衰减率为 $0.07 \%$ 每圈。Tang等 62 通过两步CVD法制备了垂直生长的氮掺杂碳纳米 管与石墨烯复合材料 $(\mathrm{N}-\mathrm{ACNT} / \mathrm{G})$ 。碳纳米管与石 墨烯片相互垂直形成类三明治的多级结构, 为电 子转移和离子扩散提供了快速通道, 而且氮掺杂
进一步提高了该碳复合材料对多硫化锂的吸附能 力。因此, N-ACNT/G 与硫的复合材料表现出了优 异的循环性能和倍率性能, 即使在 $5 C$ 大电流密度 下, 其可逆比容量仍高达 $770 \mathrm{mAh} \cdot \mathrm{g}^{-1}$ (图6b, c)。 $\mathrm{Lu}^{\text {等 }}{ }^{63}$ 首先制备了硫包覆的碳纳米纤维 ( $\mathrm{S}-\mathrm{CNFs}$ ), 然后利用静电自组装策略将石墨烯包裹在S-CNFs 表面, 得到了石墨烯硫-碳纳米纤维同轴复合材料 (G-S-CNFs)。该材料作为锂硫电池正极时, 在 $1 C$ 电 流密度下具有 $745 \mathrm{mAh} \cdot \mathrm{g}^{-1}$ 的初始比容量, 循环 1500 圈后保留 $273 \mathrm{mAh} \cdot \mathrm{g}^{-1}$, 容量衰减率为 $0.043 \%$ 每圈。Yuan等 ${ }^{64}$ 将石墨烯与离子液体 $[\mathrm{Emim}] \mathrm{BF}_{4}$ 混 合并经过两步热解得到了石墨烯支撑的富N/B碳 层(G-NBCL)。该复合材料不仅继承了石墨烯优异 的导电性, 而且其表面碳层中的 $N=B / N=B$ 结构 对多硫化锂的吸附能力明显强于吡咯氮和吡啶 氮。将 G-NBCL与硫复合作为锂硫电池正极, 在 $2 C$ 电流密度下, 循环 1500 圈之后容量衰退率仅为 $0.035 \%$ 每圈, 且在 $3 C$ 大电流密度下, 具有 480 $\mathrm{mAh} \cdot \mathrm{g}^{-1}$ 的可逆比容量。

2.3.2 石墨烯/金属化合物复合材料

最近，各种石墨烯负载的金属化合物被广泛 应用于锂硫电池正极。在该类复合材料中, 石墨烯 (a)

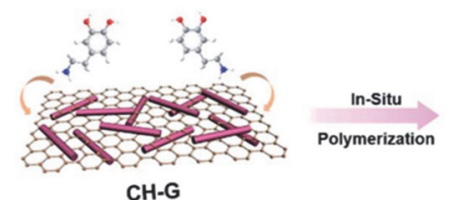

CH-G

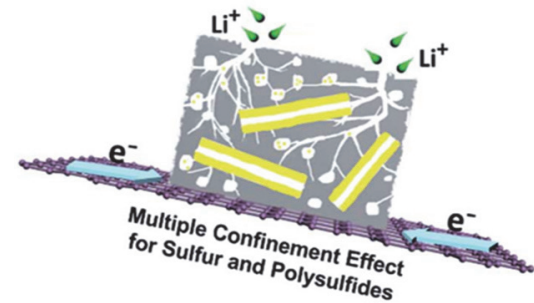

(b)

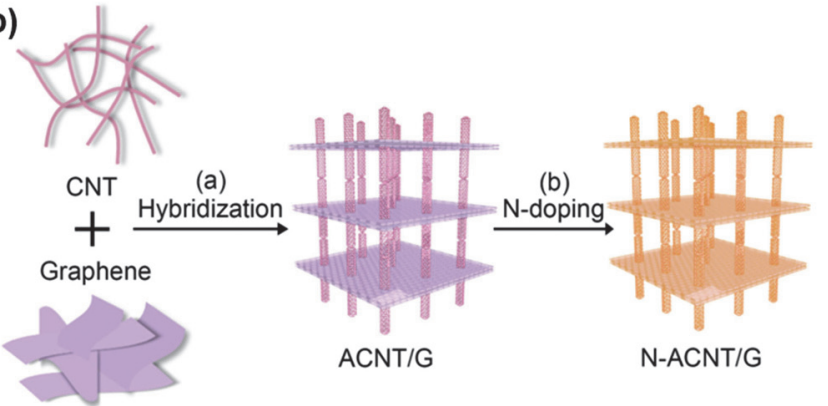

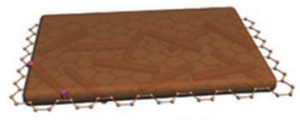

CH-G@PDA

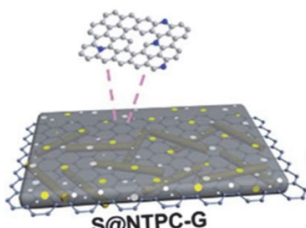

S@NTPC-G

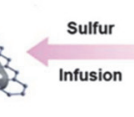

(c)

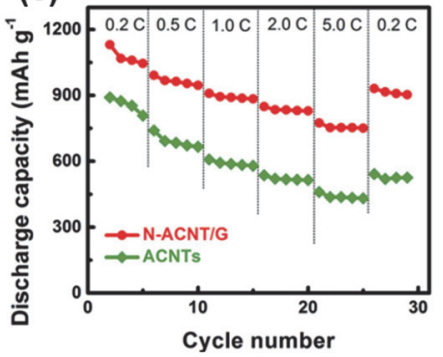

图 6 (a) NTPC-G 的合成示意图 ${ }^{61}$; (b) N-ACNT/G 的合成示意图; (c)载硫 N-ACNT/G 的倍率性能 ${ }^{62}$

Fig. 6 (a) The synthesis process of NTPC-G ${ }^{61}$; (b) The synthesis process of N-ACNT/G; (c) The rate performance of sulfur-loading N-ACNT/G ${ }^{62}$. 
不仅可作为导电网络和柔性框架来提高电极的导 电性和结构稳定性, 还可为金属化合物提供原位 成核位点, 实现其小尺寸高分散。这些金属化合物 能够暴露丰富的极性吸附位点, 将多硫化锂针定 在石墨烯基底上, 通过快速的离子与电子传输进 一步促进多硫化锂的转化, 提高锂硫电池的循环 性能和倍率性能。Yu等 ${ }^{65}$ 通过原子层沉积方法在载 硫氮掺杂石墨烯基底上生长 $\mathrm{TiO}_{2}\left(\mathrm{NG} / \mathrm{S}-\mathrm{TiO}_{2}\right)$ 。尽 管 $\mathrm{TiO}_{2}$ 对多硫化锂具有强的结合能力, 然而其较 差的导电性降低了多硫化锂的转化反应动力学, 因 此调控导电石墨烯基底上 $\mathrm{TiO}_{2}$ 的负载量对提高载 硫正极的电化学性能至关重要。最终, 原子层沉积 20 圈得到的 $\mathrm{NG} / \mathrm{S}-\mathrm{TiO}_{2}$ 复合材料实现了最优的电 化学性能。在 $1 C$ 电流密度下, 初始比容量高达 1102 $\mathrm{mAh} \cdot \mathrm{g}^{-1}$, 经过 500 圈循环后, 容量衰减率仅为 $0.024 \%$ 每圈。与金属氧化物相比, 金属硫化物、金 属氮化物、金属硒化物的电子导电性更高, 更有利 于促进多硫化锂的转化反应, 提高锂硫电池的倍 率性能。Cheng等 ${ }^{66}$ 通过水热法在还原氧化石墨烯 表面均匀负载了 $\mathrm{VS}_{2}$ 纳米片 $\left(\mathrm{VS}_{2}-\mathrm{rGO}\right)$, 然后通过 熔融扩散策略将硫封装在 $\mathrm{VS}_{2}-\mathrm{rGO}$ 的夹层中, 形成 弹性的三明治结构。这种结构不仅能够嵌入更多 的活性物质, 而且还能为 $\mathrm{Li}_{2} \mathrm{~S}$ 沉积提供充足的空间 以有效缓解体积膨胀。另外, 具有强极性且高导电 性的 $\mathrm{VS}_{2}-\mathrm{rGO}$ 基底能够有效针定多硫化锂, 并加快 界面电荷转移, 提高其转化反应动力学。硫载量高 达 $89 \%(w)$ 的 $\mathrm{VS}_{2}-\mathrm{rGO} / \mathrm{S}$ 复合材料在 $0.1 C$ 电流密度 下表现出高达 $1182 \mathrm{mAh} \cdot \mathrm{cm}^{-3}$ 的体积比容量。Sun 等 ${ }^{67}$ 将 $\mathrm{GO}$ 和 $\mathrm{NH}_{4} \mathrm{VO}_{3}$ 作为前驱体进行水热处理并 在 $\mathrm{NH}_{3}$ 气氛下热解得到了 $\mathrm{VN} / \mathrm{G}$ 复合材料。介孔 $\mathrm{VN}$ 纳米带与石墨烯相互连接形成的三维网络结构, 不 仅能够加快锂离子传输和电子转移, 还能够有效 容纳硫化锂的体积膨胀。而且, VN能通过与多硫 化锂形成 $\mathrm{S}-\mathrm{V}$ 键和 $\mathrm{Li}-\mathrm{N}$ 键有效将多硫化锂针定 在导电基底上, 进而加快其转化反应动力学。将 $\mathrm{VN} / \mathrm{G}$ 应用于锂硫电池正极时, 在 $0.2 C$ 电流密度 下, 初始比容量高达 $1461 \mathrm{mAh} \cdot \mathrm{g}^{-1}$, 在 $2 C$ 大电流密 度下, 比容量仍能保持 $956 \mathrm{mAh} \cdot \mathrm{g}^{-1}$ 。Chen等 68 结合 软模板法和肼还原法制备了中空 $\mathrm{CoSe}_{2}$ 纳米球与 还原氧化石墨烯复合材料(RGO-CoSe 2$)$ 。 $\mathrm{CoSe}_{2}$ 中 空纳米球均匀分散在石墨烯片层之间, 既能有效 阻止石墨烯堆叠, 又能有效吸附多硫化锂。而且, $\mathrm{CoSe}_{2}$ 由于其本身的金属属性能够促进电荷转移, 加快多硫化锂的电化学转化(图7)。因此, 载硫的 RGO-CoSe 2 复合材料在 $1 C$ 电流密度下具有 1037 $\mathrm{mAh} \cdot \mathrm{g}^{-1}$ 的初始比容量, 循环 400 圈后, 仍能保持
$741 \mathrm{mAh} \cdot \mathrm{g}^{-1}$ 。

\section{4 石墨烯基柔性自支撑正极}

柔性、可穿戴的电子设备的广泛应用对电化 学储能器件的设计提出了更多的要求。与传统的 电池体系相比, 柔性电池必须在经历弯曲、折叠、 拉伸挤压之后仍能恢复原始状态, 并保持原有电 化学性能。锂硫电池因其极高的理论能量密度在 轻便、柔性电池设备中具有极好的应用前景。石墨 烯具有优异的导电性、机械强度和柔韧性, 已经被 广泛应用于柔性锂硫电池正极 ${ }^{32}$ 。

Jin等 ${ }^{69}$ 首次将柔性自支撑石墨烯/硫复合材料 (GS/S) 应用于锂硫电池正极。首先, 通过硫代硫酸 钠和盐酸之间的化学反应将硫纳米颗粒均匀沉积 在石墨烯层, 然后经过真空抽滤即可得到 $\mathrm{GS} / \mathrm{S}$ 膜。 在 $0.1 C$ 电流密度下循环 100 圈之后, 该 $\mathrm{GS} / \mathrm{S}$ 柔性电 极的比容量仍保持 $600 \mathrm{mAh} \cdot \mathrm{g}^{-1}$, 容量保持率高达 $83 \%$ 。Zhou等 ${ }^{70}$ 通过CVD方法在泡沫镍上生长石墨 烯, 然后在其表面包覆一层聚二甲基硅氧烷 (PDMS), 酸洗去除镍即可得到PDMS/GF柔性薄 膜。最终将含硫浆料过滤在PDMS/GF薄膜上得到 了柔性 S-PDMS/GF电极。在石墨烯泡沫表面的 PDMS包覆层有效提高了柔性骨架的机械强度, 相 互连通的石墨烯泡沫不仅提供了电子快速传输路 径, 而且提供了足够大的空间容纳活性物质。当硫 载量高达 $10.1 \mathrm{mg} \cdot \mathrm{cm}^{-2}$ 时, 该柔性电极面积比容量 高达 $13.4 \mathrm{mAh} \cdot \mathrm{cm}^{-2}$, 而且在 $0.15 \mathrm{~A} \cdot \mathrm{g}^{-1}$ 电流密度下, 经过 1000 圈循环之后, 容量衰退率仅为 $0.071 \%$ 每 圈(图8a, b)。与石墨烯相比, 氧化石墨烯具有丰富 的含氧官能团, 更易于在水溶液中分散和组装, 并 得到与硫的柔性复合材料, 后续再经过还原即可 得到石墨烯/硫柔性电极。例如, Wang 等 ${ }^{71}$ 将硫纳

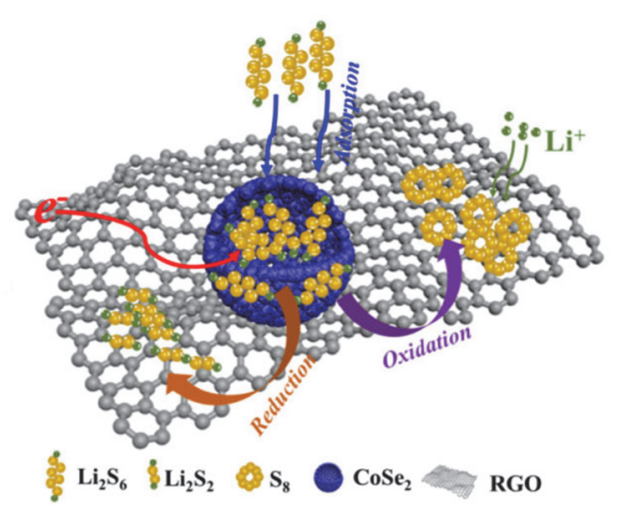

图 7 RGO-CoSe 2 在锂硫电池中的作用机制 68

Fig. 7 The function mechanism of RGO-CoSe 2 nanohybrid in Li-S batteries ${ }^{68}$.

Adapted from Tsinghua University Press. 
(a)

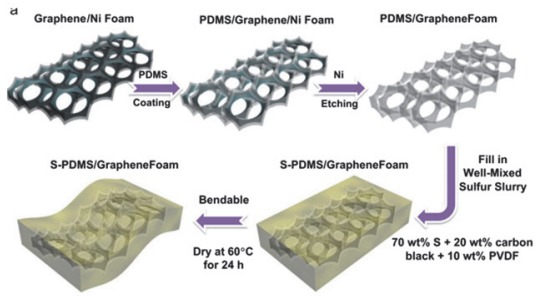

(c)

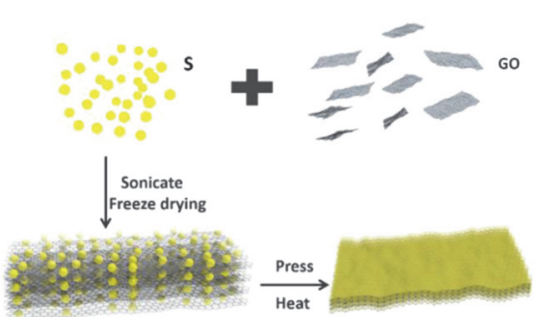

(b)

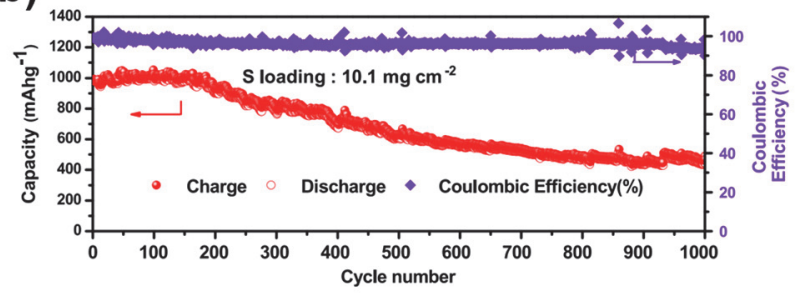

(d)

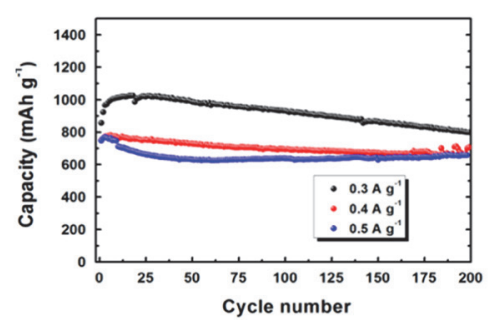

(e)

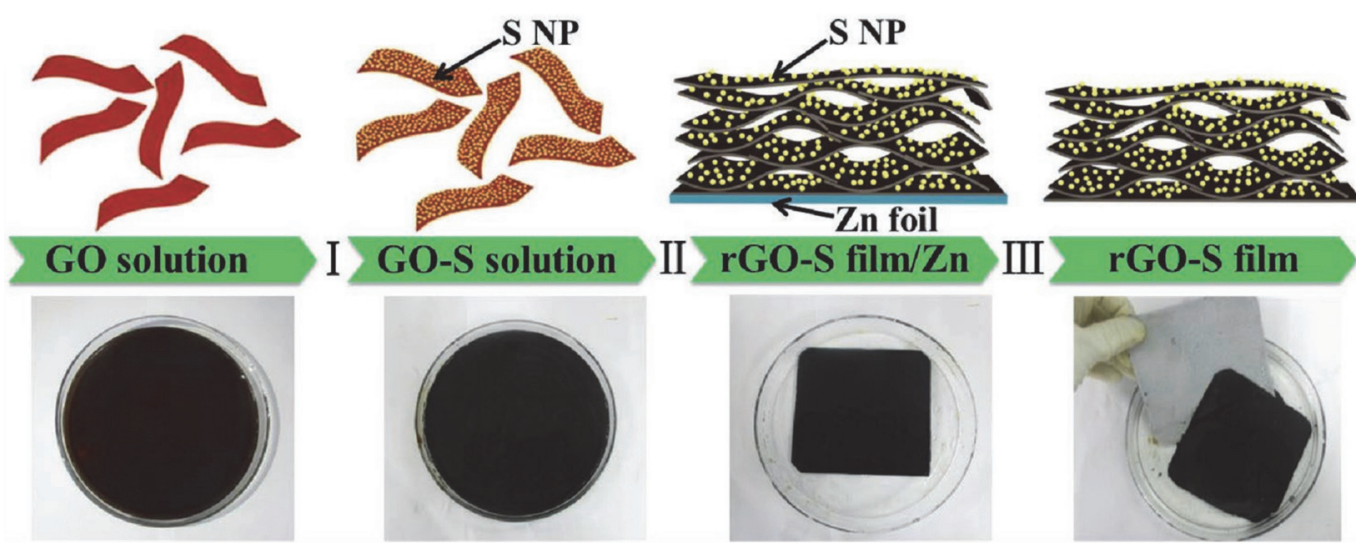

图 8 S-PDMS/GF 电极的(a)合成示意图和(b)循环性能图 ${ }^{70} ;$ S-rGO 膜的(c)合成示意图和(d) 循环性能图 ${ }^{71}$;

(e) rGO-S 复合膜的合成示意图 72

Fig. 8 (a) Synthesis schematic and (b) cycling performance of S-PDMS/GF electrodes ${ }^{70}$; (c) Synthesis schematic and (d) cycling performance of S-rGO paper ${ }^{70}$; (e) Schematic process of fabricating rGO-S composite films ${ }^{70}$. Adapted from Elsevier Science ${ }^{70,71}$ and Wiley publisher ${ }^{72}$.

米颗粒和氧化石墨烯均匀混合分散, 然后通过冷 冻干燥以及低温热处理过程制备了柔性纳米硫/石 墨烯复合膜(S-rGO)。冷冻干燥方法导致氧化石墨 烯片层褶皱弯曲, 形成丰富的大孔结构。后续高温 热处理过程中, 氧化石墨烯被还原成石墨烯, 同时 硫纳米颗粒熔融分散, 均匀包覆在石墨烯表面。该 S-rGO复合膜作为自支撑电极, 在 $0.3 \mathrm{~A} \cdot \mathrm{g}^{-1}$ 的电流 密度下, 初始比容量为 $957 \mathrm{mAh} \cdot \mathrm{g}^{-1}$, 经过 200 圈循 环, 容量仍保持 $93 \%$ (图 $8 \mathrm{c}, \mathrm{d}$ )。Cao等 ${ }^{72}$ 利用化学 反应沉积策略在氧化石墨烯表面沉积硫纳米颗粒, 尺寸仅为9-22 nm。然后, 将锌䈃浸渍到以上GO$\mathrm{S}$ 分散液中, 利用 $\mathrm{rGO} / \mathrm{GO}$ 与 $\mathrm{Zn} / \mathrm{Zn}^{2+}$ 的还原电势差, 将 $\mathrm{GO}$ 还原成 $\mathrm{rGO}$ 。在还原过程中, 电子易从锌箔 表面转移到 $\mathrm{GO}$ 片层, 导致锌箔表面离子化, 产生 的 $\mathrm{Zn}^{2+}$ 能够吸附更多带负电的 GO片, 诱导其进一 步的还原与组装(图8e)。最终剥离的 rGO-S膜具有
突出的柔性与机械强度, 因而利用该电极组装的 柔性软包电池和电缆型电池均表现出优异的电化 学性能, 在 $0.1 C$ 电流密度下, 初始比容量分别高达 1187 和 $1360 \mathrm{mAh} \cdot \mathrm{g}^{-1}$, 并且经过弯折之后容量仍然 保持稳定。

与石墨烯在常规锂硫电池正极中的应用思路 类似, 其他各种类型的碳材料、导电聚合物以及极 性材料也被引入到柔性石墨烯基电极中。石墨烯 在这些复合电极中充分发挥了其高柔韧性和高导 电性的优势 ${ }^{31,73}$ 。Shi等 ${ }^{74}$ 将氧化石墨烯和碳纳米管 混合, 经过冷冻干燥制备了氧化石墨烯/碳纳米管 气凝胶, 之后通过自蔓延燃烧被快速还原成石墨 烯/碳纳米管气凝胶。将该气凝胶同时作为硫的柔 性、致密载体以及正极和隔膜之间的夹层组装成 锂硫电池, 表现出优异的循环性能。在 $2 C$ 电流密 度下, 经过 500 圈循环之后, 比容量仍高达 445 
(a)

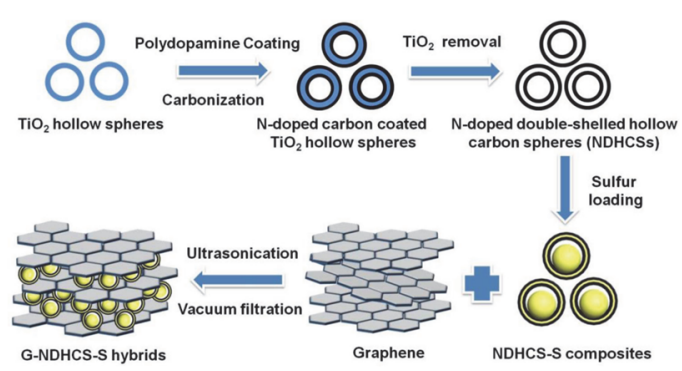

(c)

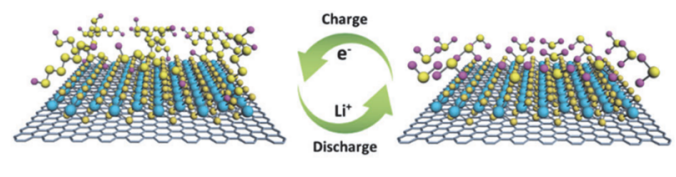

(b)

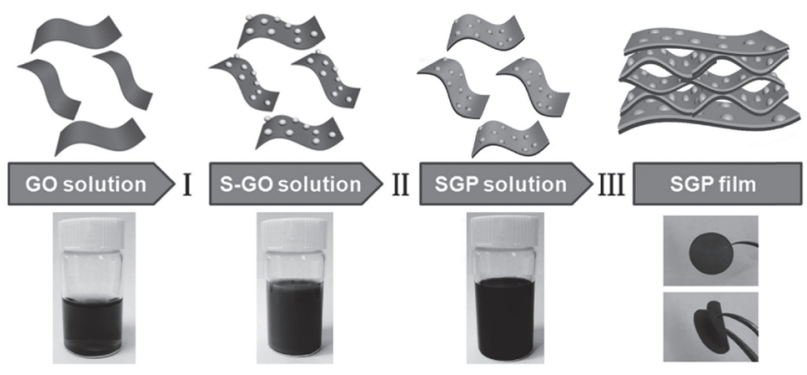

(d)

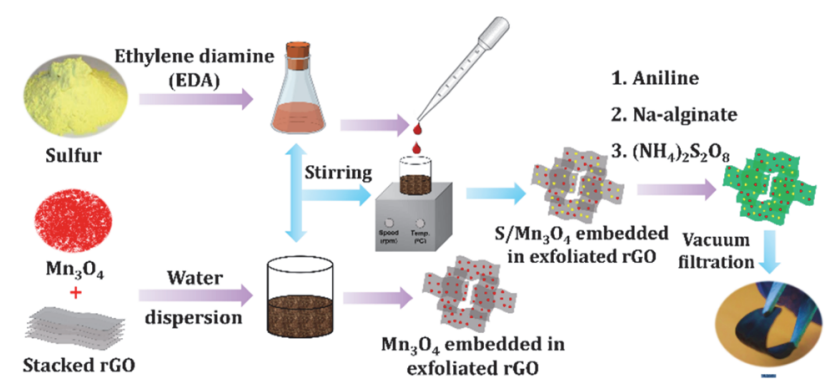

图 9 (a) G-NDHCS-S 复合膜的合成示意图 ${ }^{53}$; (b) SGP 复合膜的合成示意图 ${ }^{75}$; (c)多硫化锂在石墨烯/1T MoS 2 表面的 转化过程 ${ }^{76}$; 以及载硫石墨烯/1T $\mathrm{MoS}_{2}$ 电极的循环性能; (d) PRGO/S/ $/ \mathrm{Mn}_{3} \mathrm{O}_{4} @ \mathrm{PANI}-\mathrm{SA}$ 正极的合成示意图 ${ }^{77}$

Fig. 9 (a) Schematic illustration of the fabrication of the G-NDHCS-S hybrid paper ${ }^{53}$; (b) Schematic illustration of the

SGP cathodes ${ }^{75}$; (c) The conversion process of lithium polysulfides on a graphene surface with $1 \mathrm{~T} \mathrm{MoS}_{2}$ and cycling performance of S-loading graphene/1T $\mathrm{MoS}_{2}$ electrode ${ }^{76}$; (d) Schematic illustration of the

synthesis of the PRGO/S/Mn3 $\mathrm{O}_{4} @$ PANI-SA cathode ${ }^{77}$.

Adapted from Wiley ${ }^{53,75}$, Royal Society of Chemistry ${ }^{76}$ and ACS publications ${ }^{77}$.

$\mathrm{mAh} \cdot \mathrm{g}^{-1}$, 容量衰退率仅为 $0.06 \%$ 每圈。同时, 该电 池的体积比容量高达 $1841 \mathrm{Ah} \cdot \mathrm{L}^{-1}$ ，体积能量密度 高达 $2134 \mathrm{Wh} \cdot \mathrm{L}^{-1}$ 。Zhou等 ${ }^{53}$ 利用 $\mathrm{TiO}_{2}$ 中空球作为 模板, 多巴胺作为碳源, 合成了氮掺杂双壳中空碳 球(NDHCS), 然后通过熔融扩散策略将硫封装在 其空腔中, 得到NDHCS-S复合材料, 最后通过包 覆石墨烯、真空抽滤得到了柔性G-NDHCS-S复合 膜(图9a)。在 $2 C$ 大电流密度下, 该膜比容量高达 600 $\mathrm{mAh} \cdot \mathrm{g}^{-1}$, 并且循环 200 圈之后库伦效率仍接近 $100 \%$ 。Xiao等 ${ }^{75}$ 在沉积硫纳米颗粒的氧化石墨烯 外部包覆PEDOT:PSS, 后续经过热还原以及真空 抽滤过程得到了致密、柔性的硫-石墨烯-导电聚合 物(SGP)膜(图9b)。该膜作为锂硫电池正极, 在 $0.1 C$ 电流密度下, 体积比容量高达 $1432 \mathrm{Ah} \cdot \mathrm{L}^{-1}$; 在 $1 C$ 电流密度下, 循环 500 圈之后容量仍保持 $80 \%$ 。 $\mathrm{He}$ 等 ${ }^{76}$ 通过水热法在石墨烯表面原位生长少层 $\mathrm{MoS}_{2}$ 纳米片, 制备了自支撑的三维石墨烯/1T $\mathrm{MoS}_{2}$ 异 质结。 $1 \mathrm{~T} \mathrm{MoS}_{2}$ 具有丰富的活性位点, 有利于促进 多硫化锂转化; 通过冷冻干燥石墨烯形成的大量 孔隙结构有利于加快电解液渗透和锂离子传输; 三 维石墨烯框架和金属性 $1 \mathrm{~T} \mathrm{MoS}$ 具有优异的导电
性, 有利于加快电子转移。基于以上优点, 面载量 高达 $10 \mathrm{mg} \cdot \mathrm{cm}^{-2}$ 的柔性载硫三维石墨烯/1T $\mathrm{MoS}_{2}$ 正极在 $0.1 C$ 电流密度下, 初始比容量高达 1181 $\mathrm{mAh} \cdot \mathrm{g}^{-1}$, 经过 200 圈循环之后, 容量保持率为 $96.3 \%$ 。在 $1 C$ 大电流密度下循环 500 圈容量衰退率 仅为 $0.08 \%$ 每圈 (图9c)。Ghosh等 ${ }^{77}$ 首先通过溶液分 散混合方法得到了 $\mathrm{S} / \mathrm{Mn}_{3} \mathrm{O}_{4} /$ 石墨烯复合物, 然后 以海藻酸钠作为掺杂剂, 通过苯胺的原位聚合反 应得到聚苯胺/海藻酸钠粘结的 $\mathrm{S} / \mathrm{Mn}_{3} \mathrm{O}_{4}$ / 石 墨烯 (PRGO/S/ $/ \mathrm{Mn}_{3} \mathrm{O}_{4} @$ @PANI-SA)柔性薄膜(图9d)。聚苯 胺/海藻酸钠作为粘结剂不仅具有高的机械强度和 电子/离子导电性, 而且对多硫化锂具有一定的化 学吸附作用。以该柔性薄膜作为锂硫电池正极, 在 $5 \mathrm{~A} \cdot \mathrm{g}^{-1}$ 电流密度下, 初始比容量高达 1015 $\mathrm{mAh} \cdot \mathrm{g}^{-1}$, 经过 500 圈循环之后, 容量保持率为 $71 \%$ 。

\section{3 结论与展望}

本文总结了三维石墨烯、表面化学修饰的石 墨烯、石墨烯基复合材料以及石墨烯基柔性材料 在锂硫电池正极中的研究进展。各种载硫石墨烯 材料的电化学性能汇总于表 1 。石墨烯及其复合材 
表 1 载硫石墨烯材料的电化学性能

Table 1 Electrochemical performance of sulfur-loading graphene materials.

\begin{tabular}{|c|c|c|c|c|c|}
\hline Materials & Sulfur Content $(w / \%)$ & Current Density & Specific Capacity $/\left(\mathrm{mAh} \cdot \mathrm{g}^{-1}\right)$ & Cycle Numbers & Ref. \\
\hline Porous graphene & 66 & $1 C$ & 824 & 80 & 33 \\
\hline Unstacked double-layer templated graphene (DTG) & 64 & $10 C$ & 628 & 200 & 34 \\
\hline Hierarchical porous graphene (HPG) & 68 & $5 C$ & 656 & Initial & 35 \\
\hline Hydroxylated graphene & 50 & $2 C$ & 647 & 200 & 36 \\
\hline Functionalized holey graphene & 49 & $0.2 C$ & 429 & 300 & 37 \\
\hline Amino-functionalized reduced graphene oxide (EFG) & 60 & $0.5 C$ & 650 & 350 & 38 \\
\hline $\mathrm{N}$-doped graphene & 65.2 & $2 C$ & 492 & 1000 & 39 \\
\hline 3D B-doped graphene aerogel (BGA) & 59 & $2 C$ & 600 & 200 & 40 \\
\hline N/S co-doped graphene (A-NSG) & 72.4 & $0.2 C$ & 780 & 600 & 41 \\
\hline Co single atoms embedded in N-doped graphene $(\mathrm{Co}-\mathrm{N} / \mathrm{G})$ & 90 & $1 C$ & 681 & 500 & 50 \\
\hline Holey Fe, N co-doped graphene ( HFeNG) & 86.5 & $0.1 C$ & 1290 & Initial & 51 \\
\hline V single atoms on N-doped graphene (SAV@NG) & 80 & $0.5 C$ & 645 & Initial & 52 \\
\hline N-doped carbon channels implanted on graphene (NTPC-G) & 60 & $6 C$ & 563 & Initial & 61 \\
\hline $\mathrm{N}$ doped aligned $\mathrm{CNT} /$ graphene $(\mathrm{N}-\mathrm{ACNT} / \mathrm{G})$ & 52.6 & $1 C$ & 880 & 80 & 62 \\
\hline Coaxial graphene wrapping over sulfur-coated carbon & 33 & $1 C$ & 273 & 1500 & 63 \\
\hline \multicolumn{6}{|l|}{ nanofibers (G-S-CNFs) } \\
\hline Graphene-supported N and B rich carbon layer (G-NBCL) & 70 & $2 C$ & 301 & 1500 & 64 \\
\hline $\mathrm{TiO}_{2}$ modified N-doped graphene /sulfur composite $\left(\mathrm{NG} / \mathrm{S}-\mathrm{TiO}_{2}\right)$ & 59 & $1 C$ & 918 & 500 & 65 \\
\hline $\mathrm{VS}_{2}$-attached reduced graphene oxide sheets $\left(\mathrm{VS}_{2}-\mathrm{rGO}\right)$ & 89 & $0.1 C$ & 1013 & Initial & 66 \\
\hline VN nanoribbon/graphene composite (VN/G) & - & $1 C$ & 917 & 200 & 67 \\
\hline $\mathrm{CoSe}_{2}$ hollow nanospheres decorated rGO (RGO-CoSe $)_{2}$ & 74.7 & $1 C$ & 741 & 400 & 68 \\
\hline Graphene/PDMS Foam & - & $1.5 \mathrm{~A} \cdot \mathrm{g}^{-1}$ & 448 & 1000 & 70 \\
\hline Nano-sulfur/graphene paper (S-rGO) & 63 & $0.3 \mathrm{~A} \cdot \mathrm{g}^{-1}$ & 800 & 200 & 71 \\
\hline rGO-S composite films (rGO-S) & 56 & $0.1 C$ & 1187 & Initial & 72 \\
\hline Graphene/CNT aerogels & 50 & $2 C$ & 445 & 500 & 74 \\
\hline Graphene wrapped N-doped hollow carbon spheres-sulfur & 62 & $2 C$ & 600 & Initial & 53 \\
\hline \multicolumn{6}{|l|}{ hybrid paper (G-NDHCS-S) } \\
\hline Sulfur-graphene-conducting polymer hybrid film (SGP) & 56.4 & $1 C$ & 806 & 500 & 75 \\
\hline Graphene/1T $\mathrm{MoS}_{2}$ composite film & - & $0.1 C$ & 1181 & Initial & 76 \\
\hline Porous $\mathrm{rGO} /$ sulfur nanoparticles/ $\mathrm{Mn}_{3} \mathrm{O}_{4}$ nanoparticles with & 56.2 & $5 \mathrm{~A} \cdot \mathrm{g}^{-1}$ & 722 & 500 & 77 \\
\hline
\end{tabular}

料作为硫载体的优势在于: (1)具有高的导电性以 及结构稳定性, 能够有效提高活性物质利用率和 缓解放电过程中的体积膨胀; (2)三维网络以及丰 富的孔结构一方面可以加快电子转移和离子传 输, 另一方面可以对多硫化锂起到物理限域作用; (3)表面官能团改性和杂原子掺杂, 特别是金属单 原子掺杂, 能通过与多硫化锂之间的化学相互作 用有效吸附多硫化锂, 并加快其转化反应动力学; (4)高导电性石墨烯与强极性金属化合物复合能够 协同促进基底对多硫化锂的吸附能力与催化转化
能力；(5)具有高的柔韧性以及易于组装的特性, 非常适合构筑柔性锂硫电池电极。

尽管石墨烯在锂硫电池正极中的研究已经取 得了很大进展, 但是距离锂硫电池实用化还有很 大差距, 目前存在的主要问题有：(1)石墨烯的高 品质、大规模制备较为困难, 通过CVD或者特殊的 剥离方法得到的石墨烯品质较高, 但生产成本也 随之上升, 而通过氧化石墨烯还原策略得到的石 墨烯的物理结构性质会大打折扣; (2)高硫载量条 件下, 硫在石墨烯基底中分散不均匀甚至严重团 
聚。低液硫比条件下, 锂硫电池循环寿命明显衰 减; (3)电极内部的表界面化学反应过程尚不清晰, 石墨烯与其他材料的协同作用机理仍不明确。由 此可见, 未来在低成本制备高品质石墨烯、提高硫 载量和降低液硫比实现锂硫电池高能量密度、探 索石墨烯在锂硫电池中的储能增强作用机制等领 域亟待取得突破。研究者们可从新型石墨烯制备 技术开发、电池材料与结构设计、先进原位表征结 合理论计算等方面来突破以上难题。

\section{References}

(1) Larcher, D.; Tarascon, J. M. Nat. Chem. 2015, 7, 19. doi: 10.1038/nchem.2085

(2) Fotouhi, A.; Auger, D. J.; Propp, K.; Longo, S.; Wild, M. Renew. Sust. Energ. Rev. 2016, 56, 1008. doi: 10.1016/j.rser.2015.12.009

(3) Zhang, L.; Wang, Y.; Niu, Z.; Chen, J. Carbon 2019, 141, 400. doi: 10.1016/j.carbon.2018.09.067

(4) Liu, Y.-T.; Liu, S.; Li, G.-R.; Gao, X.-P. Adv. Mater. 2020, 33, 2003955. doi: 10.1002/adma.202003955

(5) Bruce, P. G.; Freunberger, S. A.; Hardwick, L. J.; Tarascon, J.-M. Nat. Mater. 2011, 11, 19. doi: 10.1038/nmat3191

(6) Yang, Y.; Zheng, G.; Cui, Y. Chem. Soc. Rev. 2013, 42, 3018. doi: $10.1039 / \mathrm{c} 2 \operatorname{cs} 35256 \mathrm{~g}$

(7) Zhang, M.; Yu, C.; Zhao, C.; Song, X.; Han, X.; Liu, S.; Hao, C.; Qiu, J. Energy Storage Mater. 2016, 5, 223. doi: 10.1016/j.ensm.2016.04.002

(8) Cheon, S. E.; Ko, K. S.; Cho, J. H.; Kim, S. W.; Chin, E. Y.; Kim, H. T. J. Electrochem. Soc. 2003, 150, A796. doi: $10.1149 / 1.1571532$

(9) Liu, S.; Yao, L.; Zhang, Q.; Li, L.-L.; Hu, N.-T.; Wei, L.-M.; Wei, H. Acta Phys. -Chim. Sin. 2017, 33, 2339. [刘帅, 姚路, 章琴, 李路路, 胡南滔, 魏良明, 魏浩. 物理化学学报, 2017, 33, 2339.] doi: 10.3866/PKU.WHXB201706021

(10) He, Y.; Chang, Z.; Wu, S.; Zhou, H. J. Mater. Chem. A 2018, 6, 6155. doi: $10.1039 / \mathrm{C} 8 \mathrm{TA} 01115 \mathrm{~J}$

(11) Zheng, D.; Wang, G. W.; Liu, D.; Si, J. Y.; Ding, T. Y.; Qu, D. Y.; Yang, X. Q.; Qu, D. Y. Adv. Mater. Technol. 2018, 3. doi: 10.1002/admt.201700233

(12) Deng, S.; Yan, Y.; Wei, L.; Li, T.; Su, X.; Yang, X.; Li, Z.; Wu, M. ACS Appl. Energy Mater. 2019, 2, 1266. doi: 10.1021/acsaem.8b01815

(13) Xu, Z. L.; Kim, J. K.; Kang, K. Nano Today 2018, $19,84$. doi: 10.1016/j.nantod.2018.02.006

(14) Guan, L.; Hu, H.; Li, L.; Pan, Y.; Zhu, Y.; Li, Q.; Guo, H.; Wang, K.; Huang, Y.; Zhang, M.; et al. ACS Nano 2020, 14, 6222. doi: 10.1021/acsnano.0c02294
(15) Yan, Y.; Chen, Z.; Yang, J.; Guan, L.; Hu, H.; Zhao, Q.; Ren, H.; Lin, Y.; Li, Z.; Wu, M. Small 2020, 16, 2004631. doi: $10.1002 /$ smll.202004631

(16) Chen, Z. L.; Gao, P.; Liu, Z. F. Acta Phys. -Chim. Sin. 2020, 36, 1907004. [陈召龙, 高鹏, 刘忠范. 物理化学学报, 2020, 36, 1907004.] doi: 10.3866/PKU.WHXB201907004

(17) Wang, B.; Ruan, T.; Chen, Y.; Jin, F.; Peng, L.; Zhou, Y.; Wang, D.; Dou, S. Energy Storage Mater. 2020, 24, 22. doi: 10.1016/j.ensm.2019.08.004

(18) Chen, K.; Sun, Z. H.; Fang, R. P.; Li, F.; Cheng, H. M. Acta Phys. Chim. Sin. 2018, 34, 377. [陈克, 孙振华, 方若翩, 李峰, 成会明. 物理化学学报, 2018, 34, 377.] doi: 10.3866/PKU.WHXB201709001

(19) Zhang, Y.; Gao, Z.; Song, N.; He, J.; Li, X. Mater. Today Energy 2018, 9, 319. doi: 10.1016/j.mtener.2018.06.001

(20) Sun, C.; Liu, Y.; Sheng, J.; Huang, Q.; Lv, W.; Zhou, G.; Cheng, H.-M. Mater. Horiz. 2020, 7, 2487. doi: 10.1039/d0mh00815j

(21) Wang, H.; Yang, Y.; Liang, Y.; Robinson, J. T.; Li, Y.; Jackson, A.; Cui, Y.; Dai, H. Nano Lett. 2011, 11, 2644. doi: 10.1021/n1200658a

(22) Ji, L.; Rao, M.; Zheng, H.; Zhang, L.; Li, Y.; Duan, W.; Guo, J.; Cairns, E. J.; Zhang, Y. J. Am. Chem. Soc. 2011, 133, 18522. doi: $10.1021 / \mathrm{ja} 206955 \mathrm{k}$

(23) Yang, X.; Zhang, L.; Zhang, F.; Huang, Y.; Chen, Y. S. ACS Nano 2014, 8 , 5208. doi: 10.1021/nn501284q

(24) Ning, H.; Mao, Q.; Wang, W.; Yang, Z.; Wang, X.; Zhao, Q.; Song, Y.; Wu, M. J. Alloys Compd. 2019, 785, 7. doi: 10.1016/j.jallcom.2019.01.142

(25) Zhao, Q.; Liu, J.; Li, X.; Xia, Z.; Zhang, Q.; Zhou, M.; Tian, W.; Wang, M.; Hu, H.; Li, Z.; et al. Chem. Eng. J. 2019, 369, 215. doi: 10.1016/j.cej.2019.03.076

(26) Wang, Y.; Huo. W.; Yuan, X.; Zhang, Y. Acta Phys. -Chim. Sin. 2020, 36, 1904007. [王易, 霍旺晨, 袁小亚, 张育新. 物理化学学报, 2020, 36, 1904007.] doi: 10.3866/PKU.WHXB201904007

(27) Zhang, T.; Li, C.; Wang, W.; Guo, Z.; Pang, A.; Ma, H. Acta Phys. -Chim. Sin. 2020, 36, 1905048. [张婷, 李翠翠, 王伟, 郭兆 琦, 庞爱民, 马海霞. 物理化学学报, 2020, 36, 1905048.] doi: 10.3866/PKU.WHXB201905048

(28) Li, Y.; Cai, Q.; Wang, L.; Li, Q.; Peng, X.; Gao, B.; Huo, K.; Chu, P. K. ACS Appl. Mater. Interfaces 2016, 8, 23784. doi: 10.1021/acsami.6b09479

(29) Li, Z.; Xu, R.; Deng, S.; Su, X.; Wu, W.; Liu, S.; Wu, M. Appl. Surf. Sci. 2018, 433, 10. doi: 10.1016/j.apsusc.2017.10.050

(30) Liu, D.; Zhang, C.; Zhou, G.; Lv, W.; Ling, G.; Zhi, L.; Yang, Q.-H. Adv. Sci. 2018, 5. doi: 10.1002/advs. 201700270

(31) Guo, X.; Zheng, S.; Zhang, G.; Xiao, X.; Li, X.; Xu, Y.; Xue, H.; Pang, H. Energy Storage Mater. 2017, 9, 150. doi: 10.1016/j.ensm.2017.07.006 
(32) Wang, Z.; Xu, X.; Ji, S.; Liu, Z.; Zhang, D.; Shen, J.; Liu, J. J. Mater. Sci. Technol. 2020, 55, 56. doi: 10.1016/j.jmst.2019.09.037

(33) Huang, J.-Q.; Liu, X.-F.; Zhang, Q.; Chen, C.-M.; Zhao, M.-Q.; Zhang, S.-M.; Zhu, W.; Qian, W.-Z.; Wei, F. Nano Energy 2013, 2, 314. doi: 10.1016/j.nanoen.2012.10.003

(34) Zhao, M.-Q.; Zhang, Q.; Huang, J.-Q.; Tian, G.-L.; Nie, J.-Q.; Peng, H.-J.; Wei, F. Nat. Commun. 2014, 5, 3410. doi: $10.1038 /$ ncomms 4410

(35) Tang, C.; Li, B.-Q.; Zhang, Q.; Zhu, L.; Wang, H.-F.; Shi, J.-L.; Wei, F. Adv. Funct. Mater. 2016, 26, 577. doi: 10.1002/adfm. 201503726

(36) Zu, C.; Manthiram, A. Adv. Energy Mater. 2013, 3, 1008. doi: 10.1002/aenm.201201080

(37) Chang, N.; Zhou, C. G.; Fu, H.; Zhao, Y.; Shui, J. L. Adv. Mater. Interfaces 2017, 4, 9. doi: 10.1002/admi.201700783

(38) Wang, Z.; Dong, Y.; Li, H.; Zhao, Z.; Wu, H. B.; Hao, C.; Liu, S.; Qiu, J.; Lou, X. W. Nat. Commun. 2014, 5, 5002. doi: $10.1038 /$ ncomms 6002

(39) Qiu, Y.; Li, W.; Zhao, W.; Li, G.; Hou, Y.; Liu, M.; Zhou, L.; Ye, F.; Li, H.; Wei, Z.; et al. Nano Lett. 2014, 14, 4821. doi: $10.1021 / \mathrm{n} 15020475$

(40) Xie, Y.; Meng, Z.; Cai, T.; Han, W.-Q. ACS Appl. Mater. Interfaces 2015, 7, 25202. doi: 10.1021/acsami.5b08129

(41) Xu, J.; Su, D.; Zhang, W.; Bao, W.; Wang, G. J. Mater. Chem. A 2016, 4, 17381. doi: 10.1039/c6ta05878g

(42) Hou, T. Z.; Chen, X.; Peng, H. J.; Huang, J. Q.; Li, B. Q.; Zhang, Q.; Li, B. Small 2016, 12, 3283. doi: 10.1002/smll.201600809

(43) Zhang, K.; Chen, Z.; Ning, R.; Xi, S.; Tang, W.; Du, Y.; Liu, C.; Ren, Z.; Chi, X.; Bai, M.; et al. ACS Appl. Mater. Interfaces 2019, 11, 25147. doi: 10.1021/acsami.9b05628

(44) Zhang, L.; Liu, D.; Muhammad, Z.; Wan, F.; Xie, W.; Wang, Y.; Song, L.; Niu, Z.; Chen, J. Adv. Mater. 2019, 31. 19063955. doi: 10.1002/adma.201903955

(45) Li, Y.; Lin, S.; Wang, D.; Gao, T.; Song, J.; Zhou, P.; Xu, Z.; Yang, Z.; Xiao, N.; Guo, S. Adv. Mater. 2020, 32, 1906722. doi: 10.1002/adma.201906722

(46) Li, Y.; Wu, J.; Zhang, B.; Wang, W.; Zhang, G.; Seh, Z. W.; Zhang, N.; Sun, J.; Huang, L.; Jiang, J.; et al. Energy Storage Mater. 2020, 30, 250. doi: 10.1016/j.ensm.2020.05.022

(47) Lu, C.; Chen, Y.; Yang, Y.; Chen, X. Nano Lett. 2020, 20, 5522. doi: 10.1021/acs.nanolett.0c02167

(48) Lu, C.; Fang, R.; Chen, X. Adv. Mater. 2020, 32, 1906548. doi: 10.1002/adma.201906548

(49) Zhang, Q.; Zhang, X.; Wang, J.; Wang, C. Nanotechnology 2021, 32, 032001. doi: 10.1088/1361-6528/abbd70

(50) Du, Z.; Chen, X.; Hu, W.; Chuang, C.; Xie, S.; Hu, A.; Yan, W.; Kong, X.; Wu, X.; Ji, H.; et al. J. Am. Chem. Soc. 2019, 141, 3977. doi: $10.1021 /$ jacs.8b12973

(51) Wang, Y.; Adekoya, D.; Sun, J.; Tang, T.; Qiu, H.; Xu, L.; Zhang, S.; Hou, Y. Adv. Funct. Mater. 2019, 29, 1807485. doi: 10.1002/adfm.201807485

(52) Zhou, G.; Wang, S.; Wang, T.; Yang, S.-Z.; Johannessen, B.; Chen, H.; Liu, C.; Ye, Y.; Wu, Y.; Peng, Y.; et al. Nano Lett. 2020, $20,1252$. doi: 10.1021/acs.nanolett.9b04719

(53) Zhou, G. M.; Zhao, Y. B.; Manthiram, A. Adv. Energy Mater. 2015, 5, 1402263. doi: 10.1002/aenm.201402263

(54) Chen, K.; Sun, Z.; Fang, R.; Shi, Y.; Cheng, H.-M.; Li, F. Adv. Funct. Mater. 2018, 28, 1707592. doi: 10.1002/adfm.201707592

(55) Chen, X.; Xiao, Z.; Ning, X.; Liu, Z.; Yang, Z.; Zou, C.; Wang, S.; Chen, X.; Chen, Y.; Huang, S. Adv. Energy Mater. 2014, 4, 1301988. doi: 10.1002/aenm.201301988

(56) Peng, H.-J.; Huang, J.-Q.; Zhao, M.-Q.; Zhang, Q.; Cheng, X.-B.; Liu, X.-Y.; Qian, W.-Z.; Wei, F. Adv. Funct. Mater. 2014, 24, 2772. doi: 10.1002/adfm.201303296

(57) Chen, R.; Zhao, T.; Lu, J.; Wu, F.; Li, L.; Chen, J.; Tan, G.; Ye, Y.; Amine, K. Nano Lett. 2013, 13, 4642. doi: 10.1021/n14016683

(58) Zhao, C.; Yu, C.; Zhang, M.; Yang, J.; Liu, S.; Li, M.; Han, X.; Dong, Y.; Qiu, J. J. Mater. Chem. A 2015, 3, 21842. doi: $10.1039 / \mathrm{c} 5 \mathrm{ta} 05146 \mathrm{k}$

(59) Huang, J.-Q.; Xu, Z.-L.; Abouali, S.; Garakani, M. A.; Kim, J.-K. Carbon 2016, 99, 624. doi: 10.1016/j.carbon.2015.12.081

(60) Zhang, Z.; Kong, L.-L.; Liu, S.; Li, G.-R.; Gao, X.-P. Adv. Energy Mater. 2017, 7, 1602543. doi: 10.1002/aenm.201602543

(61) Zhang, M.; Yu, C.; Yang, J.; Zhao, C.; Ling, Z.; Qiu, J. J. Mater. Chem. A 2017, 5, 10380. doi: 10.1039/c7ta01512g

(62) Tang, C.; Zhang, Q.; Zhao, M.-Q.; Huang, J.-Q.; Cheng, X.-B.; Tian, G.-L.; Peng, H.-J.; Wei, F. Adv. Mater. 2014, 26, 6100. doi: 10.1002/adma.201401243

(63) Lu, S.; Cheng, Y.; Wu, X.; Liu, J. Nano Lett. 2013, 13, 2485. doi: $10.1021 / \mathrm{n} 1400543 \mathrm{y}$

(64) Yuan, S.; Bao, J. L.; Wang, L.; Xia, Y.; Truhlar, D. G.; Wang, Y. Adv. Energy Mater. 2016, 6, 1501733. doi: 10.1002/aenm.201501733

(65) Yu, M.; Ma, J.; Song, H.; Wang, A.; Tian, F.; Wang, Y.; Qiu, H.; Wang, R. Energy Environ. Sci. 2016, 9, 1495. doi: $10.1039 / \mathrm{c} 5 \mathrm{ee} 03902 \mathrm{a}$

(66) Cheng, Z.; Xiao, Z.; Pan, H.; Wang, S.; Wang, R. Adv. Energy Mater. 2018, 8, 1702337. doi: 10.1002/aenm.201702337

(67) Sun, Z.; Zhang, J.; Yin, L.; Hu, G.; Fang, R.; Cheng, H.-M.; Li, F. Nat. Commun. 2017, 8, 14627. doi: 10.1038/ncomms 14627

(68) Chen, L.; Yang, W.; Liu, J.; Zhou, Y. Nano Res. 2019, 12, 2743. doi: $10.1007 / \mathrm{s} 12274-019-2508-3$

(69) Jin, J.; Wen, Z.; Ma, G.; Lu, Y.; Cui, Y.; Wu, M.; Liang, X.; Wu, X. RSC Adv. 2013, 3, 2558. doi: 10.1039/C2RA22808D 
(70) Zhou, G. M.; Li, L.; Ma, C. Q.; Wang, S. G.; Shi, Y.; Koratkar, N.; Ren, W. C.; Li, F.; Cheng, H. M. Nano Energy 2015, 11, 356. doi: 10.1016/j.nanoen.2014.11.025

(71) Wang, C.; Wang, X. S.; Wang, Y. J.; Chen, J. T.; Zhou, H. H.; Huang, Y. H. Nano Energy 2015, 11, 678. doi: 10.1016/j.nanoen.2014.11.060

(72) Cao, J.; Chen, C.; Zhao, Q.; Zhang, N.; Lu, Q. Q.; Wang, X. Y.; Niu, Z. Q.; Chen, J. Adv. Mater. 2016, 28, 9629. doi: 10.1002/adma.201602262

(73) Sun, L.; Kong, W. B.; Jiang, Y.; Wu, H. C.; Jiang, K. L.; Wang, J. P.; Fan, S. S. J. Mater. Chem. A 2015, 3, 5305. doi: 10.1039/c4ta06255h
(74) Shi, H. D.; Zhao, X. J.; Wu, Z. S.; Dong, Y. F.; Lu, P. F.; Chen, J.; Ren, W. C.; Cheng, H. M.; Bao, X. H. Nano Energy 2019, 60, 743. doi: 10.1016/j.nanoen.2019.04.006

(75) Xiao, P.; Bu, F.; Yang, G.; Zhang, Y.; Xu, Y. Adv. Mater. 2017, 29, 1703324. doi: 10.1002/adma.201703324

(76) He, J.; Hartmann, G.; Lee, M.; Hwang, G. S.; Chen, Y.; Manthiram, A. Energy Environ. Sci. 2019, 12, 344. doi: 10.1039/C8EE03252A

(77) Ghosh, A.; Manjunatha, R.; Kumar, R.; Mitra, S. ACS Appl. Mater. Interfaces 2016, 8, 33775. doi: 10.1021/acsami.6b11180 